\title{
Asymptotic Stability of 3D Stochastic Positive Linear Systems with Delays
}

\author{
Auras Khalid Hameed \\ Department of Computer Science, College of Science for Women University of Baghdad, Baghdad, Iraq
}

(Corresponding author's e-mail: aurask_comp@csw.uobaghdad.edu.iq)

Received: 9 April 2021, Revised: 29 June 2021, Accepted: 9 July 2021

\begin{abstract}
The article emphasizes that 3D stochastic positive linear system with delays is asymptotically stable and depends on the sum of the system matrices and at the same time independent on the values and numbers of the delays. Moreover, the asymptotic stability test of this system with delays can be abridged to the check of its corresponding 2D stochastic positive linear systems without delays. Many theorems were applied to prove that asymptotic stability for 3D stochastic positive linear systems with delays are equivalent to $2 \mathrm{D}$ stochastic positive linear systems without delays. The efficiency of the given methods is illustrated on some numerical examples.
\end{abstract}

Keywords: Asymptotic stability of stochastic systems, Stability of stochastic systems, 3D stochastic positive systems, Stochastic systems with delays, Positive systems

\section{Introduction}

Multidimensional system applications have been drastically growing in many fields such as finance, water steam, economics and medicine. The basic theory and modeling of asymptotic stability of 2D linear systems have been methodically presented by Roesser [1]. There have been numerous researches that have studied asymptotic stability of 2D systems in the literature [2-4]. One of the important issues is studying asymptotic stability for linear systems. Over the past few decades, valuable and interesting works on the asymptotic stability of 2D systems have been developed. Kaczorek [5] presented a comprehensive study concerning necessary and sufficient conditions of positive 2D systems with delays. Qin et al. [6] present new conditions for asymptotic stability of a stochastic discrete-time system using the Lyapunov function to state that the product of system matrices almost surely converges. García [7] used the center manifold theorem to prove the necessary and sufficient conditions of local asymptotic stability for a nonlinear dynamical system by reducing its order to 1D system. Control and stability problems of continuous 2D linear positive Roesser model with multiple time delays were used by Duan et al. [8] to find necessary and sufficient conditions for asymptotic stability. Liu and Caraballo [9] used the classical technique of Galerkin approximations to prove the existence of asymptotic stability of delay 2D NavierStokes system by exploiting 2 approaches. Asymptotic stability of second and third-order stochastic differential equations has been studied using a suitable Lyapunov-Krasovskii function to investigate the 0 solution [10,11]. Dami et al. [12] derived the asymptotic stability and stabilization criteria based on the Lyapunov-Krasovskii function for a class of positive fractional order of 2D linear systems with and without delays. Yang and Zheng [13] have studied and investigated the problem of sliding mode control to extend the idea of model transformation for 2D Fornasini-Marchesini local state-space model. Asymptotic stability of 2D systems and their necessary conditions have been presented using Markov semi group, Fokker-Planck equation [14-17]. Moreover, there have been many types of research that have studied asymptotic stability and its necessary conditions for systems with delays. One of the researches focuses on computing asymptotic stability of $2 \mathrm{D}$ systems with delays depends on the sum of system matrices [18], while the same topic was not studied for 3D stochastic positive linear systems with delays. This approach was became the motivation of this work. Therefore, this paper proposes many theorems to prove that asymptotic stability of 3D stochastic positive linear systems with delays depends on systems matrices and independent of the delays. Moreover, prove that the computations of asymptotic stability of $3 \mathrm{D}$ positive stochastic systems with delays can be reduced to the computation of the corresponding $2 \mathrm{D}$ stochastic positive linear systems without delays. 


\section{Methods of study}

In this study, various methods were adopted to prove asymptotic stability of $3 \mathrm{D}$ stochastic positive linear systems with delays.

The general model of 3D stochastic positive linear systems

Consider the following 3D stochastic linear systems:

$$
\begin{aligned}
x_{i+1, j+1, t+1}= & A_{1} x_{i, j, t}+A_{2} x_{i+1, j, t}+A_{3} x_{i, j+1, t}+A_{4} x_{i, j, t+1} \\
& +B_{1} u_{i, j, t}+B_{2} u_{i+1, j, t}+B_{3} u_{i, j+1, t}+B_{4} x_{i, j, t+1}+\sigma d W(t)
\end{aligned}
$$

where $\mathrm{x}_{\mathrm{i}, \mathrm{j}, \mathrm{t}} \in \mathbb{R}^{\mathrm{n}}, \mathrm{u}_{\mathrm{i}, \mathrm{j}, \mathrm{t}} \in \mathbb{R}^{\mathrm{m}}$ are input vectors at the point;

$(i, j, t), \mathrm{x}(0,0,0)=\mathrm{x}_{0,0,0}$ is a. $\mathrm{s} . \in \mathrm{L}_{2}\left(\Omega, F_{T}, \mathrm{R}_{\mathrm{nxn}}\right)$, and $\mathrm{A}_{\mathrm{k}} \in \mathbb{R}^{\mathrm{n} \times \mathrm{n} \times \mathrm{n}}, \mathrm{B}_{\mathrm{k}} \in \mathbb{R}^{\mathrm{n} \times \mathrm{m} \times \mathrm{n}}, k=1,2,3,4$.

The stochastic processes $X=\left\{X_{t}\right\}, t>0,\{W(t), t \in \tau\}$, where $i$ is the time set, $\tau=R^{+}$defined on $\left(\Omega, F_{T}, P\right)$ a complete probability space with a probability measure $P$ on $\Omega, W$ is a Brownian motion process with the parameter $\sigma, \mathrm{u}=u_{i, j, t}=0$ for $\mathrm{t} \in[-\mathrm{h}, 0), h>0$ is a constant delay, $u(t) \in R^{n}, U_{a d}=$ $L_{2}^{\mathcal{F}}\left([0, T], R^{n}\right)$ be the assumed set of admissible controls, $\sigma$ is $n \times n$ dimensional constant matrix. For every $s$ and $t, s \leq t$, belonging to $t$ the increments $W_{t}-W_{s}$ are Gaussian distributed with mean zero and variance $\sigma^{2}(t-s)$. For almost all $\omega \in \Omega$, the sample functions $t \rightarrow W_{t}(\omega)$ are uniformly continuous. The above system of Eq. (1) has the boundary conditions such that:

$x_{i, 0,0} \in \mathbb{R}^{n}, i \in \mathbb{Z}_{+} x_{0, j, 0} \in \mathbb{R}^{n}, j \in \mathbb{Z}_{+}$and $x_{0,0, t} \in \mathbb{R}^{n}, t \in \mathbb{Z}_{+}$

The system of Eq. (1) is positive if $\mathrm{x}_{\mathrm{i}, \mathrm{j}, \mathrm{t}} \in \mathbb{R}_{+}^{\mathrm{n}}, i, j, t \in \mathbb{Z}_{+}$for all $\mathrm{x}_{\mathrm{i}, 0,0} \in \mathbb{R}_{+}^{\mathrm{n}}, i \in \mathbb{Z}_{+}, \mathrm{x}_{0, \mathrm{j}, 0} \in \mathbb{R}_{+}^{\mathrm{n}}$, $j \in \mathbb{Z}_{+}, \mathrm{x}_{0,0, \mathrm{t}} \in \mathbb{R}_{+}^{\mathrm{n}}, t \in \mathbb{Z}_{+} \mathrm{andu}_{\mathrm{i}, \mathrm{j}, \mathrm{t}} \in \mathbb{R}_{+}^{\mathrm{m}}, i, j, t \in \mathbb{Z}_{+}$.

\section{Theorem 1}

The stochastic system (1) is positive if and only if:

$\mathrm{A}_{\mathrm{k}} \in \mathbb{R}_{+}^{\mathrm{n} \times \mathrm{n} \times \mathrm{n}}, \mathrm{B}_{\mathrm{k}} \in \mathbb{R}_{+}^{\mathrm{n} \times \mathrm{m} \times \mathrm{n}}, \sigma \in \mathbb{R}_{+}^{\mathrm{n} \times \mathrm{m} \times \mathrm{n}}, \mathrm{k}=1,2,3,4$

The proof is followed immediately throughout definitions and notations of $[3,15]$. The following system represents a 3D stochastic linear system, given as:

$\left[\begin{array}{l}x_{i+1, j, t}^{h} \\ x_{i, j+1, t}^{v} \\ x_{i, j, t+1}^{n}\end{array}\right]=\left[\begin{array}{ll}A_{11} A_{12} & A_{13} \\ A_{21} A_{22} & A_{23} \\ A_{31} & A_{32} A_{33}\end{array}\right]\left[\begin{array}{l}x_{i, j, t}^{h} \\ x_{i, j, t}^{v} \\ x_{i, j, t}^{n}\end{array}\right]+\left[\begin{array}{l}B_{11} \\ B_{22} \\ B_{33}\end{array}\right] u_{i, j, t}+\left[\begin{array}{l}\sigma_{11} \\ \sigma_{22} \\ \sigma_{33}\end{array}\right] \mathrm{dw}$

where $i, j, t \in \mathbb{Z}_{+}, x_{i, j, t}^{h} \in \mathbb{R}^{n_{1}}, x_{i, j, t}^{v} \in \mathbb{R}^{n_{2}}$, and $x_{i, j, t}^{n} \in \mathbb{R}^{n_{3}}$ are the state vectors (horizontal, vertical and diagonal vectors respectively) at the point $(i, j, k), \mathrm{u}_{\mathrm{i}, \mathrm{j}, \mathrm{k}} \in \mathbb{R}^{\mathrm{m}}$ are the input vectors provided that

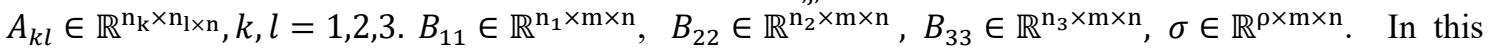
regard, the boundary conditions for the system (4) are:

$x_{0, j, t}^{h} \in \mathbb{R}^{n_{1}} j, t \in \mathbb{Z}_{+}$and $x_{i, 0, t}^{v} \in \mathbb{R}^{n_{2}}, i, t \in \mathbb{Z}_{+}, x_{i, j, 0}^{n} \in \mathbb{R}^{n_{3}} i, j \in \mathbb{Z}_{+}$

The modeling system of Eq. (4) is positive if $\mathrm{x}_{\mathrm{i}, \mathrm{j}, \mathrm{t}}^{\mathrm{h}} \in \mathbb{R}_{+}^{\mathrm{n}_{1}}, \mathrm{x}_{\mathrm{i}, \mathrm{j}, \mathrm{t}}^{\mathrm{v}} \in \mathbb{R}_{+}^{\mathrm{n}_{2}}, \mathrm{x}_{\mathrm{i}, \mathrm{j}, \mathrm{t}}^{\mathrm{n}} \in \mathbb{R}_{+}^{\mathrm{n}_{3}}$ for $i, j, t \in \mathbb{Z}_{+}$ for any nonnegative boundary conditions [5].

$\mathrm{x}_{0, \mathrm{j}, \mathrm{t}}^{\mathrm{h}} \in \mathbb{R}_{+}^{\mathrm{n}_{1}}, \mathrm{j} \in \mathbb{Z}_{+}$and $\mathrm{x}_{\mathrm{i}, 0, \mathrm{t}}^{\mathrm{v}} \in \mathbb{R}_{+}^{\mathrm{n}_{2}}, \mathrm{i}, \mathrm{t} \in \mathbb{Z}_{+} \mathrm{x}_{\mathrm{i}, \mathrm{j}, 0}^{\mathrm{n}} \in \mathbb{R}_{+}^{\mathrm{n}_{3}}, \mathrm{i}, \mathrm{j} \in \mathbb{Z}_{+}$

for all $u_{i, j, t} \in \mathbb{R}_{+}^{m}, i, j, t \in \mathbb{Z}_{+}$. 


\section{Theorem 2}

The 3D stochastic linear system of Eq. (4) is positive if and only if [19]:

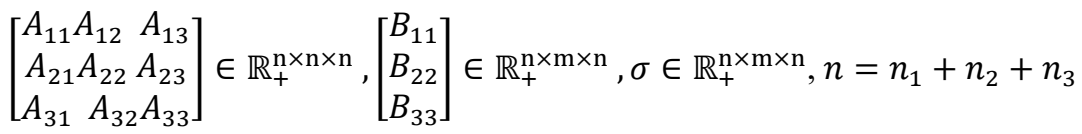

Proof: Assume that the 3D stochastic linear system of Eq. (4) is positive. Directly from the definition of positive systems of [15], the assumptions of Eq. (7) are satisfied. According to [15], the 3D stochastic linear system of Eq. (4) is negative if all its input matrices (vectors) are less than 0, which contradicts the assumptions of Eq. (5) and Theorem 1. Thus the proof is complete.

Defining

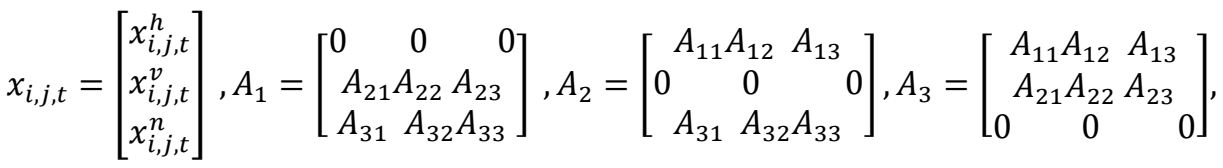

$B_{1}=\left[\begin{array}{c}0 \\ B_{22} \\ B_{33}\end{array}\right], B_{2}=\left[\begin{array}{c}B_{11} \\ 0 \\ B_{33}\end{array}\right], B_{3}=\left[\begin{array}{c}B_{11} \\ B_{22} \\ 0\end{array}\right], \sigma=\left[\begin{array}{l}\sigma_{11} \\ \sigma_{22} \\ \sigma_{33}\end{array}\right]$

rewrite the $3 \mathrm{D}$ stochastic model of Eq. (1) as below (substituting $\mathrm{A}_{1}=\mathrm{B}_{1}=0$ ):

$x_{i+1, j+1, k+1}=A_{2} x_{i+1, j, k}+A_{3} x_{i, j+1, k}+A_{4} x_{i, j, k+1}+B_{2} u_{i+1, j, k}+B_{3} u_{i, j+1, k}+B_{4} u_{i, j, k+1}+\sigma d w$

The Asymptotic stability of 3D stochastic positive linear systems without delays

The stochastic positive model of Eq. (1) is asymptotically stable if the following boundary conditions are satisfied:

$x_{i, 0,0} \in \mathbb{R}_{+}^{n}, i \in \mathbb{Z}_{+}, \quad x_{0, j, 0} \in \mathbb{R}_{+}^{n}, j \in \mathbb{Z}_{+}, x_{0,0, t} \in \mathbb{R}_{+}^{n}, \quad t \in \mathbb{Z}_{+}, \quad u_{i, j, t}=0$, for $i, j, t \in \mathbb{Z}_{+}$

$\lim _{i, j, t \rightarrow \infty} x_{i, j, t}=0$

$\forall x_{i, 0,0} \in \mathbb{R}_{+}^{n}, x_{0, j, 0} \in \mathbb{R}_{+}^{n}, x_{0,0, t} \in \mathbb{R}_{+}^{n} i, j, t \in \mathbb{Z}_{+}$

\section{Theorem 3}

For 3D stochastic positive linear system of Eq. (1), the next assumptions are equivalent [20]:

1. The stochastic model of Eq. (1) is asymptotically stable.

2. The coefficients $\hat{a}_{i}, i=1,2, \ldots, n-1$ of the characteristic polynomial of $\hat{A}, \hat{A}=A-I_{n}$, all are positive, where $A=A_{1}+A_{2}+A_{3}+A_{4}+\sigma$,

$P_{\widehat{A}}(z)=\operatorname{det}\left[I_{n} z-\hat{A}\right]=z^{n}+\hat{a}_{n-1} z^{n-1}+\cdots+\hat{a}_{1} z+\hat{a}_{0}$

3. The principal minors of the matrix

$\bar{A}=I_{n}-A=\left[\begin{array}{ccc}\bar{a}_{11} & \cdots & \bar{a}_{1 n} \\ \vdots & \cdots & \vdots \\ \bar{a}_{n 1} & \cdots & \bar{a}_{n n}\end{array}\right]$

all are positive, i.e.,

$\operatorname{det}\left[I_{n}-A\right]>0$ 
Proof: Assumption (1) is demonstrated direct from Theorem 2.1 of [4] along with imposed boundary conditions of Eq. (2). Given $n \times n$ real matrices of system (1), the characteristic polynomial defined as follows:

$z^{n}+\hat{a}_{n-1} z^{n-1}+\cdots+\hat{a}_{1} z+\hat{a}_{0}$

according to boundary conditions of Eq. (2), let us define $\wedge^{n} \hat{A}: \wedge^{n} V \rightarrow \wedge^{n} V$ which induces linear maps so that:

$\operatorname{det}\left[I_{n} z-\hat{A}\right]=z^{n}-z^{n-1} \operatorname{Tr}(\hat{A})+z^{n-2} \operatorname{Tr}\left(\wedge^{2} \hat{A}\right)-\cdots$

Conjugate $\hat{A}$ to become an upper triangular matrix with diagonal elements $\hat{a}_{i}$, so Eq. (15) becomes:

$\left(z-\hat{a}_{1}\right) \ldots\left(z-\hat{a}_{n}\right)=z^{n}-\left(\sum \hat{a}_{i}\right) z^{n-1}+\left(\sum \hat{a}_{i} \hat{a}_{j}\right) z^{n-2}-\cdots$

which is true, so Eq. (14) is true. Let $f$ be a polynomial satisfying the assumptions of the theorem, there exists a positive integern so that:

$f=\operatorname{det}\left[I_{n} z-\hat{A}\right]=z^{n}+\hat{a}_{n-1} z^{n-1}+\cdots+\hat{a}_{1} z+\hat{a}_{0}$.

According to corollary 2.1 and Theorem 2.3 of [4], the coefficients of $f$ all are positive for all $n>n_{i}, i=1,2,3,4$. Thus assumption (3) is immediately satisfied.

\section{Theorem 4}

The 3D stochastic positive linear model of Eq. (1) is unstable if at least 1 diagonal entry of the matrices:

$A=A_{1}+A_{2}+A_{3}+A_{4}+\sigma$ is $>1$.

Proof: Using lemma 2.2 of [3] we get the proof directly. As a special case, if $A_{1}=0$ the following theorem is established.

Theorem 5 For 3D stochastic positive linear system of Eq. (9), the following assumptions are equivalent:

1. The 3D stochastic positive linear system (9) is asymptotically stable.

2. The coefficients $a_{i}^{\prime}, i=0, \ldots, n-1$ of the characteristic polynomial of the matrix $A^{\prime}=A_{2}+A_{3}+$ $A_{4}+\sigma-I_{n}$ are all positive such that:

$P_{N}(z)=\operatorname{det}\left[I_{n} z-A^{\prime}\right]=z^{n}+a_{n-1}^{\prime} z^{n-1}+\cdots+a_{1}^{\prime} z+a_{0}^{\prime}$

3. The principal minors of the following matrix:

$$
-A^{\prime}=I_{n}-A_{2}-A_{3}-A_{4}-\sigma=\left[\begin{array}{ccc}
a_{11}^{\prime} & \ldots & a^{\prime}{ }_{1 n} \\
\vdots & \ldots & \vdots \\
a^{\prime}{ }_{n 1} & \ldots & a_{n n}^{\prime}
\end{array}\right]
$$

All are positive, such that:

$$
\left|a_{11}^{\prime}\right|>0, \quad\left|\begin{array}{l}
a_{11}^{\prime} a_{12}^{\prime} \\
a_{21}^{\prime} a_{22}^{\prime}
\end{array}\right|>0, \quad\left|\begin{array}{ccc}
a_{11}^{\prime} & a_{12}^{\prime} & a_{12}^{\prime} \\
a_{21}^{\prime} & a_{22}^{\prime} & a_{23}^{\prime} \\
a_{31}^{\prime} & a_{32}^{\prime} & a_{33}^{\prime}
\end{array}\right|>0,
$$

$\operatorname{det}\left[I_{n}-A_{2}-A_{3}-A_{4}-\sigma\right]>0$

Similarly, for the extended 3D stochastic positive linear system of Eq. (4), one can apply the following theorem. 


\section{Theorem 6}

Consider 3D stochastic positive model described by Eq. (4), the following statements are complement to each other:

1. The 3D stochastic positive linear model of Eq. (4) is asymptotically stable.

2. The characteristic polynomial coefficients $\left(a_{i}, i=0,1, \ldots, n-1\right)$ of the following matrix all are positive such that:

$$
\begin{aligned}
P_{R}(z) & =\operatorname{det}\left[\begin{array}{ccc}
I_{n_{1}}(z+1)-A_{11} & -A_{12} & -A_{13} \\
-\mathrm{A}_{21} & I_{n_{2}}(z+1)-A_{22} & -A_{23} \\
-A_{31} & -\mathrm{A}_{32} & I_{n_{3}}(z+1)-A_{33}
\end{array}\right] \\
& =z^{n}+a_{n-1} z^{n-1}+\cdots+a_{1} z+a_{0},
\end{aligned}
$$

where $n=n_{1}+n_{2}+n_{3}$.

All the principal minors of the matrix below are positive: $\left[I_{n_{i}}-A_{i j}\right], i, j=1, \ldots, 3$ The proof of Theorems 5 and $\mathbf{6}$ is similar to that of Theorem 3.

\section{Example 1}

$A_{0}=\left[\begin{array}{lll}0.1 & 0.1 & 0 \\ 0 & 0.2 & 0 \\ 0.1 & 0 & 0.1\end{array}\right], A_{1}=\left[\begin{array}{ccc}0.1 & 0.8 & 0 \\ 0 & 0.1 & 0 \\ 0.2 & 0 & 0.1\end{array}\right], A_{2}=\left[\begin{array}{lll}0.2 & 0.1 & 0 \\ 0 & 0.2 & 0 \\ 0.1 & 0 & 0.1\end{array}\right]$,

$A_{3}=\left[\begin{array}{lll}0.1 & 0 & 0 \\ 0 & 0.1 & 0 \\ 0.1 & 0 & 0.2\end{array}\right], \sigma=\left[\begin{array}{ccc}0.1 & 0.3 & 0.2 \\ 0 & 0.2 & 0 \\ 0.1 & 0 & 0.1\end{array}\right]$

$\hat{A}=A_{0}+A_{1}+A_{2}+A_{3}+\sigma-I_{3}=\left[\begin{array}{ccc}-0.4 & 1.3 & 0.2 \\ 0 & -0.2 & 0 \\ 0.6 & 0 & -0.4\end{array}\right]$

and

$P_{\hat{A}}(z)=\operatorname{det}\left[I_{3} z-\hat{A}\right]=\left[\begin{array}{ccc}z+0.4 & -1.3 & -0.2 \\ 0 & z+0.2 & 0 \\ -0.6 & 0 & z+0.4\end{array}\right]=z^{3}+z^{2}+0.2 z+0.008$

At this step, the matrix of Eq. (12) is:

$\bar{A}=-\hat{A}=\left[\begin{array}{ccc}0.4 & -1.3 & -0.2 \\ 0 & 0.2 & 0 \\ -0.6 & 0 & 0.4\end{array}\right]$

It is clear that the principal minors all are positive, where $\left|a_{11}\right|=0.4,\left|a_{22}\right|=\left|\begin{array}{cc}0.4 & -1.3 \\ 0 & 0.2\end{array}\right|=$ 0.08 , and $|\bar{A}|=0.056$. Thus, all the conditions of Theorem 3 are completely satisfied. Moreover, the general 3D stochastic model of Eq. (20) is asymptotically stable.

\section{Example 2}

To demonstrate the issue of asymptotic stability of the 3D stochastic positive linear system presented in Eq. (4), apply assumptions of Theorem 6 as follows:

$$
\begin{aligned}
& A_{11}=\left[\begin{array}{ccc}
0.2 & 0.1 & 0.2 \\
0 & 0.2 & 0.2 \\
0 & 0 & 0.4
\end{array}\right], A_{12}=\left[\begin{array}{cc}
0 & 0 \\
0.1 & 0 \\
0.4 & 0.5
\end{array}\right], A_{13}=\left[\begin{array}{c}
0 \\
0.1 \\
0
\end{array}\right], A_{21}=\left[\begin{array}{lll}
0 & 0 & 0.4 \\
0 & 0 & 0.2
\end{array}\right], \\
& A_{22}=\left[\begin{array}{ll}
0 & 0.1 \\
0 & 0.4
\end{array}\right], A_{23}=\left[\begin{array}{c}
0 \\
0.4
\end{array}\right], A_{31}=\left[\begin{array}{lll}
0 & 0 & 0.1
\end{array}\right], A_{32}=\left[\begin{array}{ll}
0 & 0
\end{array}\right], A_{33}=0.5
\end{aligned}
$$


Using Eq. (19) to get:

$$
=\left|\begin{array}{cccccr}
\mathrm{Z}+0.8 & -0.1 & -0.2 & 0 & 0 & 0 \\
0 & z+0.8 & -0.2 & -0.1 & 0 & -0.1 \\
0 & 0 & z+0.6 & -0.4 & -0.5 & 0 \\
0 & 0 & -0.4 & z+1 & -0.1 & 0 \\
0 & 0 & -0.2 & 0 & z+0.6 & -0.4 \\
0 & 0 & -0.1 & 0 & 0 & z+0.5
\end{array}\right|
$$

$=z^{6}+4.4 z^{5}+7.97 z^{4}+7.55 z^{3}+3.99 z^{2}+1.1268 z+0.1296$.

The principal minors are:

$M_{1}=0.8, \quad M_{2}=\left|\begin{array}{cc}0.8 & -0.1 \\ 0 & 0.8\end{array}\right|=0.64, \quad M_{3}=\left|\begin{array}{ccc}0.8 & -0.1 & -0.2 \\ 0 & 0.8 & -0.2 \\ 0 & 0 & 0.6\end{array}\right|=0.384$,

$M_{4}=\left|\begin{array}{cccc}0.8 & -0.1 & -0.2 & 0 \\ 0 & 0.8 & -0.2 & -0.1 \\ 0 & 0 & 0.6 & -0.4 \\ 0 & 0 & -0.4 & 1\end{array}\right|=0.2816, M_{5}=\left|\begin{array}{ccccc}0.8 & -0.1 & -0.2 & 0 & 0 \\ 0 & 0.8 & -0.2 & -0.1 & 0 \\ 0 & 0 & 0.6 & -0.4 & -0.5 \\ 0 & 0 & -0.4 & 1 & -0.1 \\ 0 & 0 & -0.2 & 0 & 0.6\end{array}\right|=0.0998$

and

$M_{6}=\left|\begin{array}{cccccc}0.8 & -0.1 & -0.2 & 0 & 0 & 0 \\ 0 & 0.8 & -0.2 & -0.1 & 0 & -0.1 \\ 0 & 0 & 0.6 & -0.4 & -0.5 & 0 \\ 0 & 0 & -0.4 & 1 & -0.1 & 0 \\ 0 & 0 & -0.2 & 0 & 0.6 & -0.4 \\ 0 & 0 & -0.1 & 0 & 0 & 0.5\end{array}\right|=0.036096$.

All are positive.

From the previous results; it is clear that the above 3D stochastic positive linear system of Eq. (21) is asymptotically stable since all conditions of Theorem $\mathbf{6}$ are completely satisfied.

Remark 1 Convergence and asymptotic stability of the 3D stochastic linear equations are intimately related to the signs of eigenvalues.

3D stochastic general models with delays

Consider the 3D stochastic positive linear system with delays which has the following form:

$x_{i+1, j+1, t+1}=\sum_{k=0}^{r}\left(A_{k}^{0} x_{i-k, j-k, t-k}+A_{k}^{1} x_{i+1-k, j-k, t-k}+A_{k}^{2} x_{i-k, j+1-k, t-k}+A_{k}^{3} x_{i-k, j-k, t+1-k}\right)+\sigma \mathrm{dw}$,

$i, j, t \in \mathbb{Z}_{+}$where $x_{i, j, t} \in \mathbb{R}_{+}^{n}, A_{k}^{b} \in \mathbb{R}_{+}^{n \times n \times n}, k=0,1, \ldots, r ; b=0, \ldots, 3$

Define

$\bar{x}_{i, j, t}=\left[\begin{array}{c}x_{i, j, t} \\ x_{i-1, j-1, t-1} \\ x_{i-2, j-2, t-2} \\ \vdots \\ x_{i-r, j-r, t-r}\end{array}\right] \in \mathbb{R}^{\bar{N}}, \bar{N}=(r+1) n$

and the matrices; 


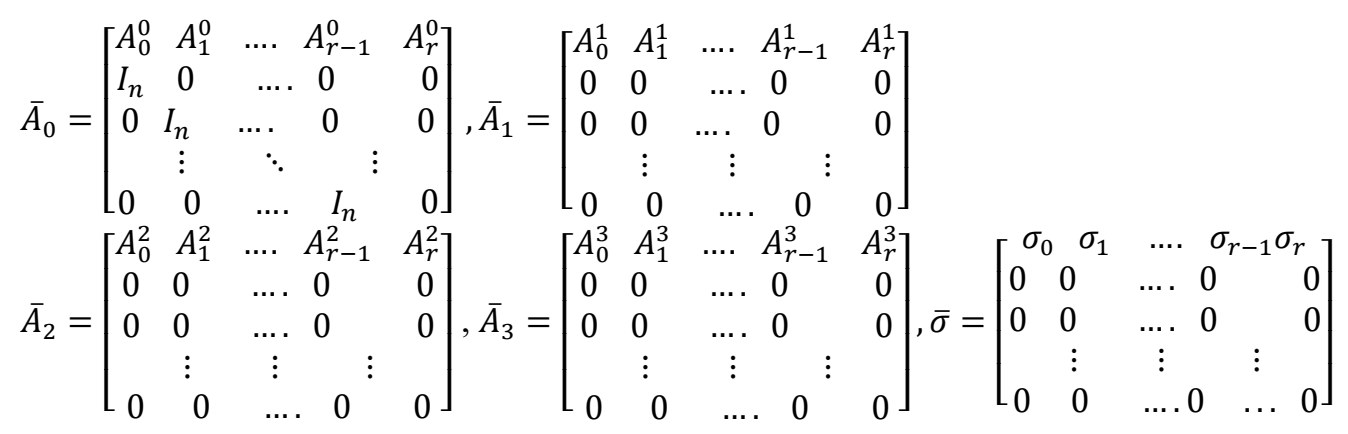

Eq. (22) becomes:

$\bar{x}_{i+1, j+1, t+1}=\bar{A}_{0} \bar{x}_{i, j, t}+\bar{A}_{1} \bar{x}_{i+1, j, t}+\bar{A}_{2} \bar{x}_{i, j+1, t}+\bar{A}_{3} \bar{x}_{i, j, t+1}+\bar{\sigma} d w \quad i, j, t \in \mathbb{Z}_{+}$

Thus, the general stochastic model of Eq. (22) has been condensed to a general model with higher dimension but without delays. When Theorem 1 is applied to Eq. (25), we can get the following result.

\section{Theorem 7}

The general 3D stochastic modeling system of $r$ delays in state as illustrated in Eq. (22) is positive if and only if $A_{k}^{b} \in \mathbb{R}_{+}^{n \times n}, k=0,1, \ldots, r, b=0,1,2,3$ or, equivalently, $\bar{A}_{b} \in \mathbb{R}_{+}^{\bar{N} \times \bar{N} \times \bar{N}}$ for $b=0,1,2,3$.

Proof: In order to prove asymptotic stability of 3D stochastic positive linear system described in Eq. (25), it is sufficient to satisfy any condition of Theorem 3. In the same manner, one can obtain an extended general model of the 3D stochastic positive linear system that has the form:

$$
\begin{aligned}
& x_{i+1, j+1, t+1}= \\
& \sum_{k=0}^{r_{1}+\sum_{f=0}^{r_{2}} \sum_{l=0}^{r_{3}}\left(A^{0} x_{i-k, j-f, t-l}+\right.}+A^{1} x_{i-k+1, j-f, t-l}+A^{2} x_{i-k, j-f+1, t-l}+ \\
& A^{3} x_{i-k, j-f, t-l+1} \quad+\sigma d w, i, j, t \in \mathbb{Z}_{+},
\end{aligned}
$$

where $x_{i, j, t} \in \mathbb{R}_{+}^{n}, A^{b} \in \mathbb{R}_{+}^{n \times n \times n}, b=0,1,2,3$.

\section{D Stochastic system with delays}

Consider the following 3D stochastic positive modeling system with $r$ delays written as:

$\left[\begin{array}{l}x_{i+1, j, t}^{h} \\ x_{i, j+1, t}^{v} \\ x_{i, j, t+1}^{n}\end{array}\right]=\sum_{l=0}^{r} A_{l}\left[\begin{array}{l}x_{i-l, j, t}^{h} \\ x_{i, j-l, t}^{v} \\ x_{i, j, t-l}^{n}\end{array}\right]+\sigma \mathrm{dw} \quad i, j, t \in \mathbb{Z}_{+}$

where $x_{i, j, t}^{h} \in \mathbb{R}_{+}^{n_{1}}, x_{i, j, t}^{v} \in \mathbb{R}_{+}^{n_{2}}, x_{i, j, t}^{n} \in \mathbb{R}_{+}^{n_{3}}$ are the state vectors at $(i, j, t)$ and

$A_{l}=\left[\begin{array}{ccc}A_{11}^{l} & A_{12}^{l} & A_{12}^{l} \\ A_{21}^{l} & A_{22}^{l} & A_{23}^{l} \\ A_{31}^{l} & A_{32}^{l} & A_{33}^{l}\end{array}\right], l=0,1, \ldots r$.

Define the vectors

$$
\begin{aligned}
\bar{x}_{i, j, t}^{h} & =\left[\begin{array}{c}
x_{i, j, t}^{h} \\
x_{i-1, j, t}^{h} \\
\vdots \\
x_{i-r, j, t}^{h}
\end{array}\right], \bar{x}_{i, j, t}=\left[\begin{array}{c}
x_{i, j, t}^{v} \\
x_{i, j-1, t}^{v} \\
\vdots \\
x_{i, j-r, t}^{v}
\end{array}\right] \\
\bar{x}_{i, j, t}^{n} & =\left[\begin{array}{c}
x_{i, j, t}^{n} \\
x_{i, j, t-1}^{n} \\
\vdots \\
x_{i, j, t-r}^{n}
\end{array}\right]
\end{aligned}
$$


Eq. (27) can be written in the form:

$\left[\begin{array}{l}\bar{x}_{i+1, j, t}^{h} \\ \bar{x}_{i, j+1, t}^{v} \\ \bar{x}_{i, j, t+1}^{n}\end{array}\right]=A\left[\begin{array}{l}\bar{x}_{i, j, t}^{h} \\ \bar{x}_{i, j, t}^{v} \\ \bar{x}_{i, j, t}^{n}\end{array}\right]+\sigma \mathrm{dw} \quad i, j, t \in \mathbb{Z}_{+}$

where the matrix $\mathrm{A}$ is defined as:

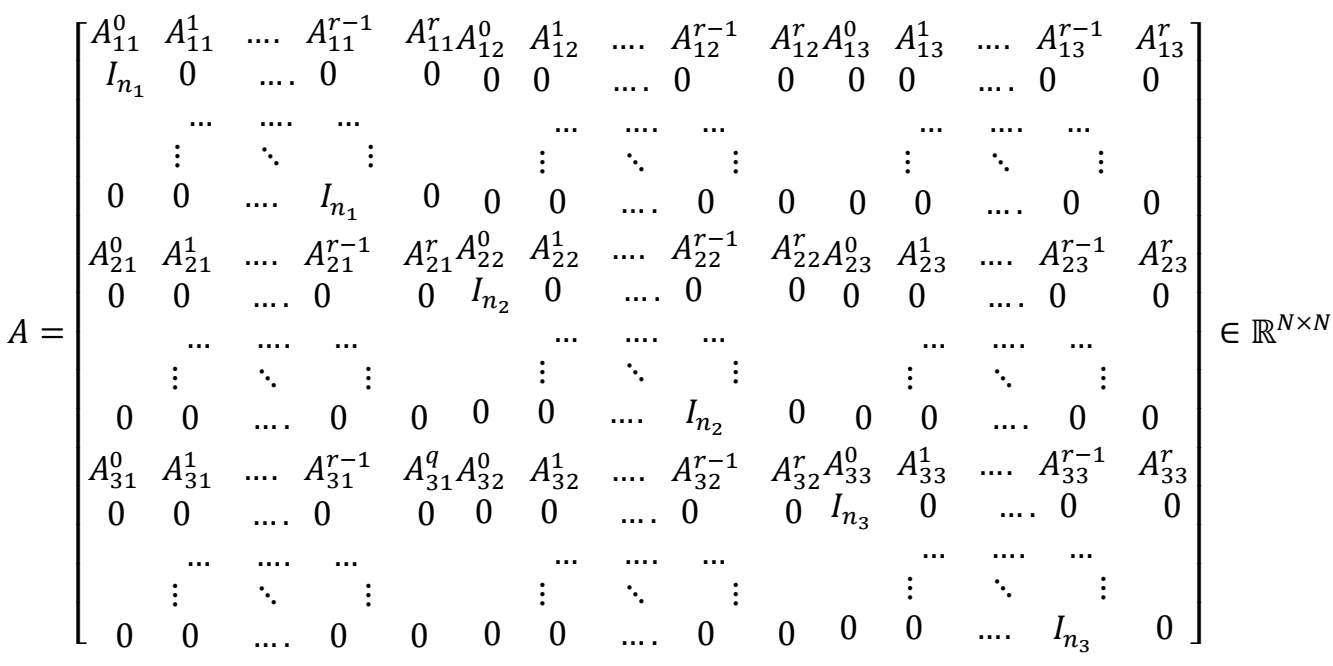

$N=(r+1)\left(n_{1}+n_{2}+n_{3}\right)$.

Thus, the 3D stochastic positive linear system without delays has been achieved.

\section{Theorem 8}

The 3D stochastic linear system with $r$ delays described in Eq. (27) is positive if and only if $A_{k} \in \mathbb{R}_{+}^{\left(n_{1}+n_{2}+n_{3}\right) \times\left(n_{1}+n_{2}+n_{3}\right)}$ for each $k=0,1, \ldots r$, which is equivalent to $A \in \mathbb{R}_{+}{ }^{N \times N \times N}$.

Proof: To prove the asymptotic stability of 3D stochastic positive model of Eq. (27), the fulfillment of any condition of Theorem $\mathbf{6}$ is sufficient.

In the same manner, 3D stochastic model of Eq. (25) can be extended to the form:

$\left[\begin{array}{l}x_{i+1, j, t}^{h} \\ x_{i, j+1, t}^{v} \\ x_{i, j, t+1}^{n}\end{array}\right]=\sum_{l=0}^{r_{1}} \sum_{k=0}^{r_{2}} \sum_{r=0}^{r_{3}} A\left[\begin{array}{l}x_{i-l, j-k, t-r}^{h} \\ x_{i-l, j-k, t-r}^{v} \\ x_{i-l, j-k, t-r}^{n}\end{array}\right]+\sigma \mathrm{dw} \quad i, j, t \in \mathbb{Z}_{+}$,

where $x_{i, j, t}^{h} \in \mathbb{R}^{n_{1}}, x_{i, j, t}^{v} \in \mathbb{R}^{n_{2}}, x_{i, j, t}^{n} \in \mathbb{R}^{n_{3}}$ are horizontal, vertical and diagonal vectors at $(i, j, t)$, where $A \in \mathbb{R}^{\left(n_{1}+n_{2}+n_{3}\right) \times\left(n_{1}+n_{2}+n_{3}\right)}$.

Relation between asymptotic stabilities of 3D stochastic positive linear systems with delays and 2D stochastic positive systems without delays

In this section, many theorems will be used to prove asymptotic stability of the reduced 2D stochastic positive linear systems without delays.

\section{Theorem 9}

The 3D stochastic positive linear system of the form:

$x_{i+1, j+1, t+1}=A_{0} x_{i, j, t}+\sum_{k=1}^{r} A_{k} x_{i-k, j-k, t-k}+\sigma d w$,

is asymptotically stable where $A_{k} \in \mathbb{R}^{n \times n \times n}$, if and only if the 2D stochastic positive linear system 
$x_{i+1, j+1}=A x_{i, j}+\sigma d w, A=\sum_{k=0}^{r} A_{k}$

is also asymptotically stable, where $, k=0,1, \ldots, r$. The proof is similar to that of Theorem 3 .

\section{Theorem 10}

For 3D stochastic positive general modeling system described by Eq. (22), next assumptions are equivalent:

1. The general 3D stochastic positive linear system is asymptotically stable.

2. The principal minors of the following matrix:

$I_{n}-\hat{A}=I_{n}-\sum_{k=0}^{r} A_{k}^{0}+A_{k}^{1}+A_{k}^{2}+A_{k}^{3}+\sigma$

all are positive.

3. The coefficients of the matrix:

$\hat{A}^{\prime}-I_{n}=\left(\sum_{k=0}^{r} A_{k}^{0}+A_{k}^{1}+A_{k}^{2}+A_{k}^{3}+\sigma\right)-I_{n}$,

$\operatorname{det}\left[I_{n}(z+1)-\hat{A}^{\prime}\right]=z^{n}+\hat{a}_{n-1}^{\prime} z^{n-1}+\cdots+\hat{a}^{\prime}{ }_{1} z+\hat{a}^{\prime}{ }_{0}$,

all are positive.

Proof: We have previously shown that 3D stochastic positive linear system without delays is asymptotically stable if and only if so is the corresponding 2D stochastic positive linear system. Applying Theorem 9, the 2D stochastic positive linear model with delays is asymptotically stable if and only if the corresponding 2D stochastic positive model without delays is also asymptotically stable. Using Theorem $\mathbf{3}$, the assumptions above will be satisfied.

Remark 2 The 3D stochastic positive general model of Eq. (22) is asymptotically stable if and only if the matrix $\hat{A}$ is a Schur matrix.

\section{Theorem 11}

The 3D stochastic positive system of Eq. (27) satisfies the following statements:

1. The positive $3 \mathrm{D}$ stochastic model is asymptotically stable.

2. All the principal minors of the following matrix are positive

$I_{n}-\hat{A}=I_{n}-\sum_{k=0}^{r}\left[\begin{array}{lll}A_{11}^{k} A_{12}^{k} & A_{12}^{k} \\ A_{21}^{k} & A_{22}^{k} & A_{23}^{k} \\ A_{31}^{k} & A_{32}^{k} & A_{33}^{k}\end{array}\right]$

3. All coefficients of the characteristic polynomial of the matrix $\hat{A}^{\prime}-I_{n}$,

$\operatorname{det}\left[I_{n}(z+1)-\hat{A}^{\prime}\right]=z^{n}+\hat{a}_{n-1}^{\prime} z^{n-1}+\cdots+\hat{a}_{1} z+\hat{a}_{0}$,

all are positive. The proof is similar to Theorem $\mathbf{1 0}$.

\section{Example 3}

To examine the applicability of Theorem 10 on Eq. (22), let us define the following matrices:

$$
\begin{aligned}
& A_{0}^{0}=\left[\begin{array}{ccc}
0.13 & 0.02 & 0 \\
0.1 & 0 & 0 \\
0 & 0.13 & 0.1
\end{array}\right], A_{1}^{0}=\left[\begin{array}{ccc}
0.02 & 0 & 0 \\
0 & 0.01 & 0.1 \\
0.2 & 0.1 & 0
\end{array}\right], A_{0}^{1}=\left[\begin{array}{ccc}
0.2 & 0 & 0 \\
0 & 0.2 & 0 \\
0.03 & 0.1 & 0.1
\end{array}\right], A_{1}^{1}=\left[\begin{array}{ccc}
0.3 & 0 & 0 \\
0 & 0.1 & 0 \\
0 & 0.1 & 0.2
\end{array}\right] \\
& A_{0}^{2}=\left[\begin{array}{lll}
0 & 0.1 & 0 \\
0 & 0.1 & 0 \\
0.12 & 0.1 & 0
\end{array}\right], A_{1}^{2}=\left[\begin{array}{ccc}
0.1 & 0 & 0 \\
0 & 0.1 & 0 \\
0 & 0.02 & 0.01
\end{array}\right], A_{0}^{3}=\left[\begin{array}{cccc}
0.01 & 0.4 & 0 \\
0 & 0.02 & 0 \\
0 & 0.1 & 0.17
\end{array}\right], A_{1}^{3}=\left[\begin{array}{ccc}
0.19 & 0 & 0 \\
0.05 & 0.11 & 0 \\
0 & 0.1 & 0.13
\end{array}\right],
\end{aligned}
$$




$$
\sigma=\left[\begin{array}{lll}
0 & 0.3 & 0 \\
0.09 & 0 & 0 \\
0.2 & 0 & 0.1
\end{array}\right] .
$$

Using Eqs. (35) and (36) one can get

$$
\begin{aligned}
& {\left[\begin{array}{ccc}
z+0.95 & -0.82 & 0 \\
-0.24 & z+0.64 & 0.1 \\
-0.55 & -0.75 & z+0.81
\end{array}\right]} \\
& \operatorname{det}\left[I_{n}(z+1)-\hat{A}\right]=z^{3}+2.4 z^{2}+1.7741 z+0.4495
\end{aligned}
$$

Thus, all principal minors are positive; $M_{1}=0.95, M_{2}=0.4112, M_{3}=0.11635$.

\section{Conclusions}

General conclusions remarks to achieve asymptotic stability of 3D stochastic positive linear systems with delays can be summarized as follows:

1. Asymptotic stability of 3D stochastic positive linear systems with delays depends on the sum of the systems matrices only, while it is independent on the number and values of the delays for that system.

2. The asymptotic stability test of the $3 \mathrm{D}$ stochastic positive linear systems with delays can be reduced to the test of asymptotic stability of the corresponding stochastic positive 2D linear system without any delays.

3. Numerical examples can be used to support the application of each theorem.

4. All theorems can be applied to 3D stochastic Hybrid systems.

\section{References}

[1] RP Roesser. A discrete state-space model for linear image processing. IEEE Trans. Automat. Contr. $1975 ; 20,1-10$.

[2] E Fornasini and G Marchesini. State-space realization theory of two-dimensional filters. IEEE Trans. Automat. Contr. 1976; 21. 484-92.

[3] A Hmamed, A Benzaouia, MA Rami and F Tadeo. Memoryless control to drive states of delayed continuous-time systems within the nonnegative orthant. In: Proceedings of the $17^{\text {th }}$ World Congress. The International Federation of Automatic Control Seoul, Korea. 2008, p. 6-11.

[4] TJ Laffey, R Loewy and H Smigoc. Power series with positive coefficients arising from the characteristic polynomials of positive matrices. Math. Ann. 2012; 364, 687-707.

[5] T kaczorek. Independence of asymptotic stability of positive 2D linear Systems with delays of their delays. Int. J. Appl. Math. Comput. Sci. 2009; 2, 255-61.

[6] Y Qin, M Cao and B Anderson. Lyapunov criterion for stochastic systems and its applications in distributed computation. IEEE Trans Automat Contr. 2020; 65, 546-60.

[7] AG García. Necessary and sufficient condition for asymptotic stability of nonlinear ordinary differential equation. Nonlinear Sci. Lett. 2018; 8, 223-34.

[8] Z Duan, I Ghous, B Wang and J Shen. Necessary and sufficient stability criterion and stabilization for positive 2-D continuous-time systems with multiple delays. Asian J. Contr. 2019; 21, 1355-66.

[9] L Liu and T Caraballo. Analysis of a stochastic 2D Navier-Stokes model with infinite delay. $J$. Dynam. Differ. Equat. 2019; 31, 2249-74.

[10] $\mathrm{O}$ Tunc and $\mathrm{C}$ Tunc. On the asymptotic stability of solutions of stochastic differential delay equations of second order. J. Taibah Univ. Sci. 2019; 13, 875-82.

[11] AM Mahmood and C Tunc. Asymptotic stability of solutions for a kind of third-order stochastic differential equations with delays. Miskolc Math. Notes 2019; 20, 381-93.

[12] L Dami, M Benhayoun and A Benzaouia. Stabilization of positive 2D fractional-order continuous time systems with delays. Circ. Syst Signal Process. 2019; 38, 1962-81.

[13] R Yang and WX Zheng. Model transformation based sliding mode control of discrete-time 2D Fornasini-Marchesini systems. J. Franklin Inst. 2019; 356, 2463-73.

[14] RD McAllister and JB Rawlings. Stochastic lyapunov functions and asymptotic syability in probability. Technical Report Number 2020-02, Texas - Wisconsin - California Control Consortium, Available at: https://sites.engineering.ucsb.edu/ jbraw/jbrweb-archives/techreports/twccc-2020-02.pdf 
[15] AN Vargas and JBR Val. Asymptotic stability of linear stochasticsystems with delay driven by a Bernoulli process. In: Proceedings of the $10^{\text {th }}$ Portuguese Conference on Automatic Control, Funchal, Portugal. 2012, p. 157-61.

[16] J Čermák and L Nechvátal. On exact and discretized stability of a linear fractional delay differential equation. Appl. Math. Lett. 2020; 105, 106296.

[17] G Liu, H Qi, Z Chang and X Meng. Asymptotic stability of a stochastic May mutualism system. Comput. Math. Appl. 2020; 3, 735-45.

[18] T Kaczorek. Positive 1D and 2D systems. Springer Verlag, London, 2001.

[19] T Kaczorek. Asymptotic stability of positive 2D linear systems with delays. Bull. Pol. Acad. Sci. Tech. Sci. 2009; 57, 133-8.

[20] T Kaczorek. Asymptotic stability of positive 2D linear systems. In: Proceedings of the $14^{\text {th }}$ Scientific Conference on Computer Applications in Electrical Engineering, Poznan, Poland. 2009, p. 1-11. 